SUPPORTING INFORMATION

\title{
Molecular Weight Dependence of the Viscosity of Highly Entangled Polyisobutylene
}

Timothy C. Ransom ${ }^{1,2}$, Debjani Roy ${ }^{1,3,4}$, Judit E. Puskas ${ }^{5}$, Gabor Kaszas ${ }^{6}$, and C. Michael Roland ${ }^{1, *}$

${ }^{1}$ Naval Research Laboratory, Chemistry Division, Code 6105, Washington DC 20375

${ }^{2}$ ASEE Postdoctoral Fellow

${ }^{3}$ National Research Council Postdoctoral Fellow

${ }^{4}$ current affiliation: US Patent and Trademark Office, Alexandria, VA 22314

${ }^{5}$ The Ohio State University, Dept. Food, Agricultural, and Biological Eng., Wooster, OH 44691

${ }^{6}$ PolyFiberMatrix LLC, Akron, OH 44325

* roland@nrl.navy.mil 
To determine the molecular weight of the fraction used for rheological measurements, a $0.1 \mathrm{~g} / \mathrm{L}$ THF solution was injected into an SEC instrument consisting of a Waters 515 HPLC Pump, Waters 2487 Dual Absorbance UV Detector, Wyatt OPTILAB DSP Interferometric Refractometer, Wyatt DAWN EOS multi-angle light scattering detector, Wyatt ViscoStar viscometer, Wyatt QELS quasi-elastic light scattering instrument, Waters 717 plus autosampler, and 6 Styragel ${ }^{\circledR}$ columns (HR6, HR5, HR4, HR3, HR1 and H0.5). The columns were thermostated at $35{ }^{\circ} \mathrm{C}$, with THF continuously distilled from $\mathrm{CaH} 2$ used as the mobile phase at a flow rate of $1 \mathrm{~mL} / \mathrm{min}$. Results were analyzed using ASTRA software (Wyatt Technology) taking $d n / d c=0.108$. Note the PIB was close to the exclusion limit of the SEC instrument. The SEC traces are shown in the figure below. Note the absence of second peaks, which affirms there is no "tail" in the molecular weight distribution.

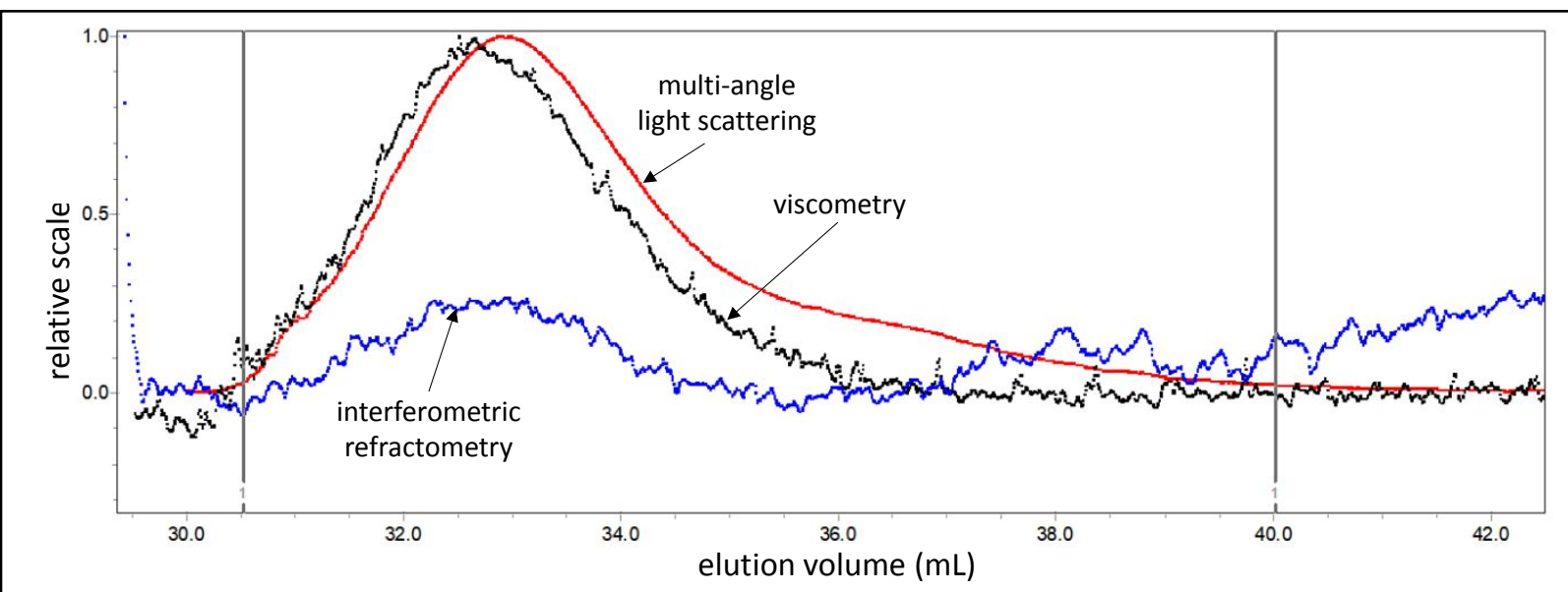

Figure S1. SEC traces of PIB for which $M_{w}=4.5 \times 10^{6} \mathrm{Da}$.

If the creep data in Figs. 2 and 3 did not correspond to steady-state, the zero-shear viscosity would be underestimated. To corroborate the result for $160^{\circ} \mathrm{C}$ the approximation method due to Ninomiya ${ }^{25}$ was applied to the data. There are two equations derived from the definition of the compliance:

$$
\eta_{0}=\left(J(t) / t-J_{s}^{0} / t\right)^{-1}
$$

with the intercept of a plot of $J(t) / t$ versus reciprocal time yielding $\eta_{0}$, and

$$
\eta_{0}=\left(\lim _{1 / t \rightarrow 0}\left[\frac{J(t)}{t} \frac{d \log J(t)}{d \log t}\right]\right)^{-1}
$$

These two essentially equivalent methods are shown in Figure S2, yielding $\eta_{0}=34 \pm 1 \mathrm{MPa}$ s. This is in good agreement with the viscosity directly obtained from the reciprocal slope of the creep curve, thus verifying that steady-state had been attained in the measurements. 


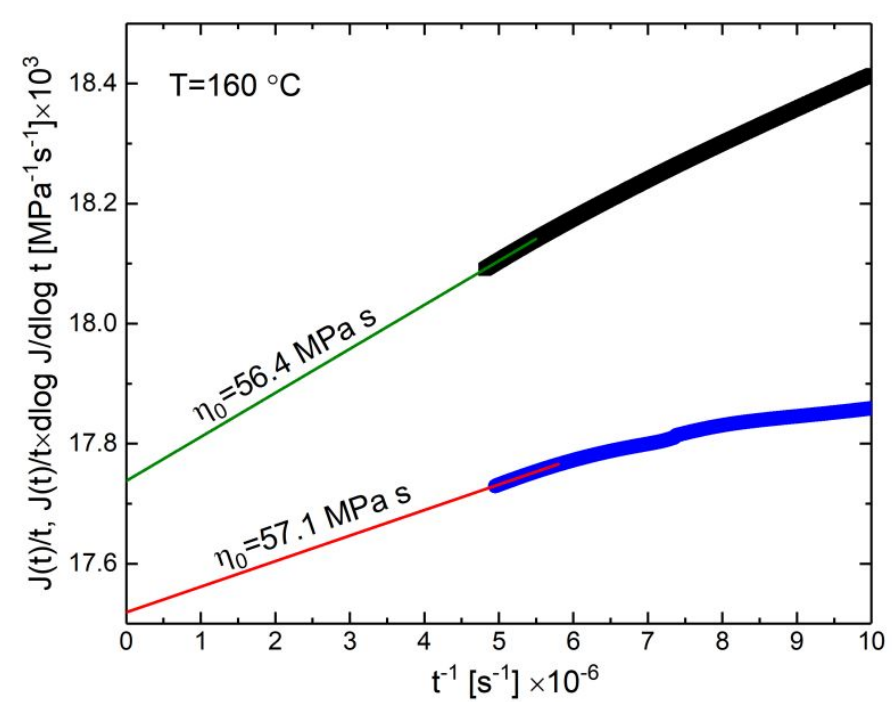

Figure S2. Approximation to steady-state viscosity [ref. 25]. The solid lines are linear fits to last 37 hours of creep, the intercept of which yields the indicated values of $\eta_{0}$.

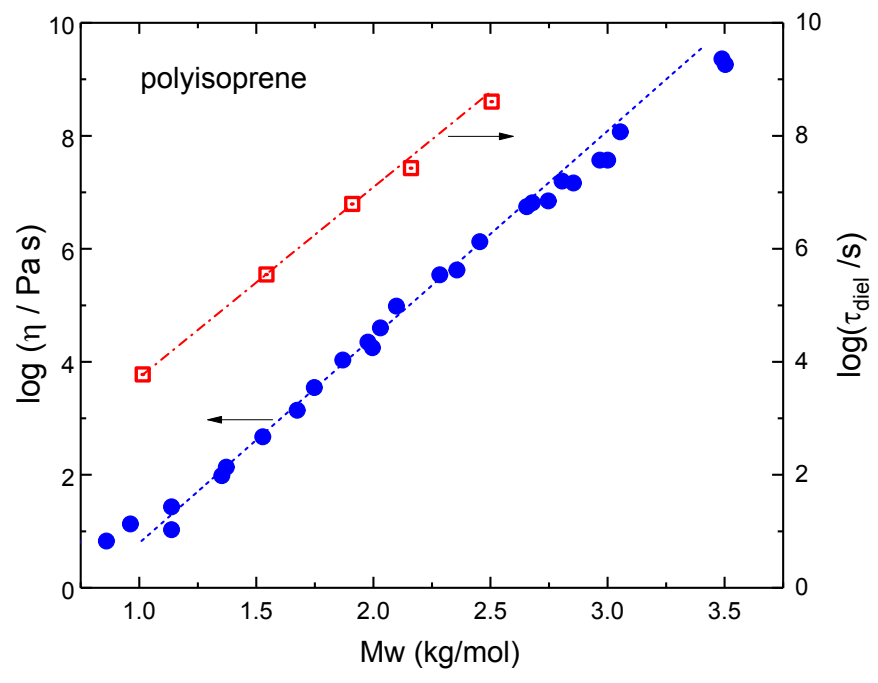

Figure S3. Viscosities [ref. 15] and dielectric normal mode relaxation times [ref. 45] for polyisoprene, with lines intended to represent the behavior prior to the pure reptation regime. 